\title{
Clinical Utility of Different Approaches for Detection of Late Pseudoprogression in Glioblastoma With O-(2-[ $\left.{ }^{18} \mathrm{~F}\right]$ Fluoroethyl)-L-Tyrosine PET
}

\author{
Olivia Kertels, MD, * Milena I. Mihovilovic, MSc, $\uparrow$ Thomas Linsenmann, MD, $\neq$ Almuth F. Kessler, MD, $\neq$ \\ Johannes Tran-Gia, PhD, $\uparrow$ Malte Kircher, MD, $\uparrow$ Joachim Brumberg, $M D, \uparrow$ \\ Camelia Maria Monoranu, MD, $\S$ Samuel Samnick, PhD, $†$ Ralf-Ingo Ernestus, MD, $\neq$ Mario Löhr, MD, $\neq$ \\ Philipp T. Meyer, MD,// and Constantin Lapa, MD ${ }^{\dagger}$
}

\begin{abstract}
Purpose: PET/CT using O-(2-[ $\left.{ }^{18} \mathrm{~F}\right]$ fluoroethyl)-L-tyrosine $\left({ }^{18} \mathrm{~F}-\mathrm{FET}\right)$ has proven valuable in differentiating tumor recurrence and progression from therapy-induced changes. This study aimed to investigate the diagnostic performance of several analytic approaches in the setting of suspected late pseudoprogression (PsP) in glioblastoma multiforme (GBM).

Methods: Retrospective analysis of tumor recurrence was performed in 36 patients with histopathologically confirmed GBM and suspicion of recurrence/disease progression more than 12 weeks from cessation of irradiation based on MRI and Response Assessment in Neuro-Oncology working group criteria. For differentiation of late PsP from true tumor recurrence, images were analyzed semiquantitatively employing tumor-to-brain ratios using 5 different approaches for tumor and normal brain reference region definition, respectively. Histopathology and/or clinical and imaging follow-up served as reference. Respective areas under the receiver operating characteristic curve were compared. Results: ${ }^{18}$ F-FET PET was able to reliably differentiate PsP from true tumor progression with areas under the receiver operating characteristic curve ranging from 0.80 to 0.88 (all $P<0.01$ ). Irrespective of the approach chosen, the classification differences between the applied methods were not significant (all $P>0.05$ ), albeit approaches focusing on voxels with the highest uptake tended to perform superior.
\end{abstract}

Received for publication December 18, 2018; revision accepted April 11, 2019. From the *Institute of Diagnostic Radiology, and Departments of $\uparrow$ Nuclear Medicine and \$Neurosurgery, University Hospital Würzburg; §Department of Neuropathology, Institute of Pathology, University of Würzburg, Würzburg; and ||Department of Nuclear Medicine, Medical Center-University of Freiburg, Faculty of Medicine, University of Freiburg, Freiburg, Germany.

P.T.M. and C.L. contributed equally to this work.

Informed consent was obtained from all individual participants included in the study. All procedures involving human participants were in accordance with the ethical standards of the institutional and/or national research committee and with the 1964 Helsinki Declaration and its later amendments or comparable ethical standards.

Conflicts of interest and sources of funding: none declared.

Authorship: Conception and design: O.K., M.I.M., T.L., A.F.K., P.T.M., C.L. Development of methodology: O.K., M.K., J.B., S.S., M.L., P.T.M., C.L. Acquisition of data: O.K., M.I.M., M.K., J.B., C.M.M., C.L. Analysis and interpretation of data: O.K., M.I.M., J.T.-G., J.B., C.M.M., C.L. Writing, review and/or revision of the manuscript: all authors. Administrative, technical, or material support: J.T.-G., S.S., R.-I.E., M.L., P.T.M. Supervision: R.-I.E., M.L., P.T.M., C.L.

Correspondence to: Constantin Lapa, MD, Department of Nuclear Medicine, University Hospital Würzburg, Oberdürrbacher Str. 6, 97080 Würzburg, Germany. E-mail: Lapa c@ukw.de.

Supplemental digital content is available for this article. Direct URL citation appears in the printed text and is provided in the HTML and PDF versions of this article on the journal's Web site (www.nuclearmed.com).

Copyright $\odot 2019$ The Author(s). Published by Wolters Kluwer Health, Inc. This is an open-access article distributed under the terms of the Creative Commons Attribution-Non Commercial-No Derivatives License 4.0 (CCBY-NC-ND), where it is permissible to download and share the work provided it is properly cited. The work cannot be changed in any way or used commercially without permission from the journal.

ISSN: 0363-9762/19/4409-0695

DOI: $10.1097 /$ RLU.0000000000002652
Conclusions: Irrespective of the analytical approach, ${ }^{18} \mathrm{~F}$-FET PET is a robust tool for detection of late PsP with only minor differences between different analytical approaches. However, methodological standardization and harmonization are needed to ensure comparability between different centers.

Key Words: FET, glioma, PET, region of interest, volume of interest

(Clin Nucl Med 2019;44: 695-701)

$\mathrm{P}$ ET using O- $\left(2-\left[{ }^{18} \mathrm{~F}\right]\right.$ fluoroethyl $)$-L-tyrosine $\left({ }^{18} \mathrm{~F}-\mathrm{FET}\right)$ as a marker of amino acid transport is an established tool in brain tumor imaging, including grading, ${ }^{1-3}$ delineation of tumor extent ${ }^{4}$ biopsy guidance, ${ }^{5,6}$ prognostication, ${ }^{7-9}$ treatment monitoring, ${ }^{10-12}$ and differentiation of nonspecific posttherapeutic changes from tumor recurrence. ${ }^{13-15}$

In daily routine, differentiation of viable tumor from treatmentrelated changes is predominantly established by determination of tracer uptake of both the tumor and normal brain tissue and subsequent calculation of tumor-to-brain ratios (TBRs). However, various approaches for deriving the respective values have been published in the literature, hampering comparability of data between different studies and sites. ${ }^{16,17}$ Given the need for prospective multicenter trials (eg, to strengthen clinical evidence for the utility of PET-based imaging in glioma or for use of ${ }^{18} \mathrm{~F}$-FET for treatment decisions and planning ${ }^{18}$ ), a well-founded selection of the most accurate approach and subsequent methodological standardization is of high relevance.

As a first step in this direction, the aim of the present study was to assess the diagnostic performance of several analytic approaches in the setting of suspected late pseudoprogression (PsP) in glioblastoma multiforme (GBM).

\section{MATERIALS AND METHODS}

\section{Subjects}

This retrospective analysis was performed using data from a previously published cohort of 36 patients (22 males and 14 females; aged 24-75 years; mean age, $54 \pm 14$ years) with histopathologically confirmed GBM. All patients were referred to ${ }^{18} \mathrm{~F}$-FET PET/CT because of MRI-based suspicion of recurrence/disease progression, as determined by the Response Assessment in Neuro-Oncology (RANO) working group criteria. ${ }^{14}$ Patients were identified consecutively and selected according to the initial scan date, which ranged from April 2010 to August 2016. The interval between cessation of radiation therapy and subsequent PET imaging was more than 12 weeks in all cases. The local ethics committee of the University of Würzburg approved this retrospective analysis of routinely acquired data. 


\section{Tracer Synthesis and PET}

Synthesis of ${ }^{18} \mathrm{~F}$-FET was performed in-house at the University Hospital of Würzburg with methods previously described, ${ }^{19}$ using a GE TRACERlab FX-FN synthesis module (GE Medical Systems, Uppsala, Sweden).

All patients fasted for at least 12 hours before PET imaging. ${ }^{20}$ Twenty minutes after intravenous injection of ${ }^{18}$ F-FET $(217 \pm 13 \mathrm{MBq})$, the patients were scanned using an integrated PET/CT scanner (Biograph mCT 64; Siemens Healthineers, Knoxville, Tenn). PET emission data were collected in 3-dimensional mode using a $200 \times 200$ matrix for 10 minutes.

Subsequent CT scans for attenuation correction were acquired using a low-dose protocol (CARE Dose 4D; $80 \mathrm{mAs}$; $120 \mathrm{kV}$; matrix, $512 \times 512$; 3-mm slice thickness; increment, $30 \mathrm{~mm} / \mathrm{s}$; rotation time, $0.5 \mathrm{~second}$; pitch index, 0.8). PET images were reconstructed iteratively (TrueX; 3 iterations; 24 subsets; Gaussian filtering, $2 \mathrm{~mm}$; decay, attenuation, and scatter correction) using dedicated manufacturer software (syngo MI.PET/CT; Siemens Healthineers).

\section{Image Analysis}

Tumor-to-brain ratios were determined according to 4 previously described $^{13,14,21,22}$ and additionally proposed semiquantitative analysis methods. Irrespective of the approach, the transaxial slice with the highest amino acid uptake was chosen for further analysis. SUVs of both the tumor and normal brain tissue, as well as respective TBR, were determined as follows:

1) Kebir et $\mathrm{al}^{13}$ first selected the transaxial slice with the highest tracer accumulation of the tumor. Next, normal brain uptake was determined by a region of interest (ROI) of 50-mm diameter placed on the contralateral hemisphere in an area of normal-appearing brain tissue including white and gray matter. $^{20} \mathrm{SUV}_{\text {mean }}$ of the tumor was defined by a 2-dimensional autocontouring process using a TBR of at least 1.6. For tumor areas with the highest uptake ( $\left.\mathrm{SUV}_{16 \mathrm{~mm}}\right)$, a circular ROI with a diameter of $16 \mathrm{~mm}$ was centered on maximal tumor uptake. Mean TBR and maximum TBR $\left(\mathrm{TBR}_{\text {mean }}\right.$ and $\left.\mathrm{TBR}_{16 \mathrm{~mm}}\right)$ were calculated by dividing the $\mathrm{SUV}_{\text {mean }}$ within these 2 tumor ROIs by the $\mathrm{SUV}_{\text {mean }}$ of normal brain. ${ }^{13}$

2) Rapp et $\mathrm{al}^{22}$ used an approach similar to (1), with the only difference of $\mathrm{SUV}_{\max }$ being the voxel with the highest amino acid uptake. ${ }^{22}$

3) Mihovilovic et $\mathrm{al}^{14}$ determined $\mathrm{SUV}_{\max }$ as the voxel with the maximum tracer uptake and $\mathrm{SUV}_{10 \mathrm{~mm}}$ as the $\mathrm{SUV}_{\text {mean }}$ in an ROI with $10-\mathrm{mm}$ diameter centered on this voxel. For derivation of $\mathrm{TBR}_{\max }$ and $\mathrm{TBR}_{10 \mathrm{~mm}}$, a second region with $50-\mathrm{mm}$ diameter including white and gray matter was selected in an area of normal-appearing brain tissue on the contralateral hemisphere of the same slice. ${ }^{14}$

4) Pöpperl et $\mathrm{al}^{21}$ calculated the tumor $S U V_{\max }$ by using the voxel with the maximum FET uptake. In the same slice, the $\mathrm{SUV}_{\text {mean }}$ within $80 \%$ and $70 \%$ isocontour ROIs was assessed $\left(\mathrm{SUV}_{80 \%}\right.$ and $\mathrm{SUV}_{70 \%}$, respectively). For TBR calculation, the background uptake was derived from the mean of the $70 \%$ and $80 \%$ isocontour ROIs mirrored to the contralateral hemisphere. ${ }^{21}$

5) The use of a crescent-shaped region for assessment of normal brain uptake is used in clinical routine at some centers (see also Unterrainer et $\mathrm{al}^{16}$ ). We further refined this approach ("crescent shaped") by using a crescent-shaped volume of interest (VOI) (composed of 3 ROIs) positioned on 3 transaxial slices on the level of the basal ganglia (striatum/capsula interna). The ROIs include cortical and subcortical gray and white matter by delineating the outer rim of the cortex from frontal to occipital and the inner cortical to subcortical interface at the depth of the sulci and the capsula interna, so that the putamen is included. Furthermore, the ventricles as well as enlarged outer cerebrospinal fluid spaces or possible structural lesions (eg, infarctions, although not present in the current sample) are carefully excluded. Tumor $\mathrm{SUV}_{\text {mean }}$ and $\mathrm{SUV}_{\text {max }}$ were calculated as defined by Rapp

TABLE 1. Overview of Region Definitions Used for the Different Analytical Approaches

\begin{tabular}{|c|c|c|}
\hline & Tumor Region (Target) & Normal Brain Region (Reference) \\
\hline Kebir et a $1^{13}$ & $\begin{array}{l}\mathrm{SUV}_{16 \mathrm{~mm}} \text { : Mean uptake within a circular ROI of } \\
16-\mathrm{mm} \text { diameter centered on the voxel with } \\
\text { maximal tumor uptake } \\
\mathrm{SUV}_{\text {mean }}^{1} \text { : Isocontour ROI applied to the transaxial } \\
\text { slice with maximum tumor uptake including all } \\
\text { voxels exceeding a predefined TBR of }>1.6\end{array}$ & $\begin{array}{l}\text { ROI of } 50-\mathrm{mm} \text { diameter placed on the } \\
\text { contralateral hemisphere including } \\
\text { white and gray matter }\end{array}$ \\
\hline Rapp et $\mathrm{a}^{22}$ & $\begin{array}{l}\mathrm{SUV}_{\text {max }}^{2} \text { : Voxel with maximum uptake } \\
\text { SUV mean: Isocontour ROI applied to the transaxial slice } \\
\text { with maximum tumor uptake including all } \\
\text { voxels exceeding a predefined TBR of }>1.6\end{array}$ & $\begin{array}{l}\text { ROI of } 50-\mathrm{mm} \text { diameter placed on the } \\
\text { contralateral hemisphere including } \\
\text { white and gray matter }\end{array}$ \\
\hline Mihovilovic et $\mathrm{al}^{14}$ & $\begin{array}{l}\mathrm{SUV}_{\max }^{2}: \text { Voxel with maximum uptake } \\
\mathrm{SUV}_{10 \mathrm{~mm}}: \text { Circular ROI of } 10 \text {-mm diameter } \\
\text { centered on voxel with maximal tumor }\end{array}$ & $\begin{array}{l}\text { ROI of } 50 \text {-mm diameter placed on } \\
\text { the contralateral hemisphere including } \\
\text { white and gray matter }\end{array}$ \\
\hline Pöpperl et $\mathrm{al}^{21}$ & $\begin{array}{l}\mathrm{SUV}_{\max } \text { : Voxel with maximum uptake } \\
\text { SUV }_{70 \%} \text { and } \mathrm{SUV}_{80 \%}: 70 \% \text { and } 80 \% \text { isocontour } \\
\text { ROIs applied to the transaxial slice } \\
\text { with maximum tumor uptake, respectively }\end{array}$ & $\begin{array}{l}\text { Mean value of the } 70 \% \text { and } 80 \% \text { isocontour } \\
\text { ROIs mirrored onto the contralateral hemisphere }\end{array}$ \\
\hline
\end{tabular}

Superscripts 1 and 2 denote pairs of identical analytical approaches. 
et al. ${ }^{22}$ In addition, we used a $90 \%$ isocontour covering the lesion on the transaxial slice with the highest uptake ( $\left.\mathrm{SUV}_{90 \%}\right)$.

Table 1 and Figure 1 provide an illustration of the various analytic approaches.

\section{Diagnosis of True Progression}

Diagnosis of true tumor progression was based on histopathologic proof, clinical deterioration, and/or further radiological progression in a follow-up MRI at least 4 weeks after the initial assessment. ${ }^{23}$

Histopathologic diagnosis of tumor recurrence was established according to standard morphologic criteria including detection of highly cellular areas composed of pleomorphic tumor cells with nuclear atypia and brisk mitotic activity as well as prominent microvascular proliferation and palisading necrosis as supporting but not mandatory criteria.

In cases without solid GBM manifestations, criteria to distinguish between true progression and PsP included higher cellularity compared with normal brain tissue, presence of cellular pleomorphism, nuclear atypia and mitoses, and elevated proliferation index (Ki67) and p53 nuclear expression in glial cells (glial fibrillary acidic protein). The density of macrophages (CD68) as potential confounder of proliferation index was also assessed.

In contrast, the diagnosis of PsP was applied in cases of negative histopathology, stable clinical conditions for at least 6 months (with no treatment changes within this time period), or stabilization/ regression of the contrast-enhancing lesions at follow-up MRI (at least 4 weeks following initial assessment), respectively. ${ }^{24}$

\section{Statistical Analysis}

Descriptive statistics for patient characteristics were reported as mean $\pm \mathrm{SD}$, median, and range. $t$ Test for independent samples was used to compare means between clinical conditions. The diagnostic performances of the different analytical approaches were assessed by calculating and comparing the area under the receiver operating characteristic (ROC) curves (R Package pROC version 1.13.0 [R Foundation for Statistical Computing, Vienna, Austria], according to DeLong et $\mathrm{al}^{25}$ ). Optimal cutoff values for the various TBR measures indicative of true progression (as opposed to PsP) were determined by using the Youden index for cutoff selection. All statistical tests were performed 2 -sided, and $P<0.05$ was considered to indicate statistical significance.

\section{RESULTS}

\section{Patient Characteristics}

This retrospective study included 36 subjects with histologically proven GBM. Patients underwent imaging while receiving first-line treatment consisting of temozolomide-based radiochemotherapy with adjuvant temozolomide according to Stupp et $\mathrm{al}^{26}(\mathrm{n}=34)$,

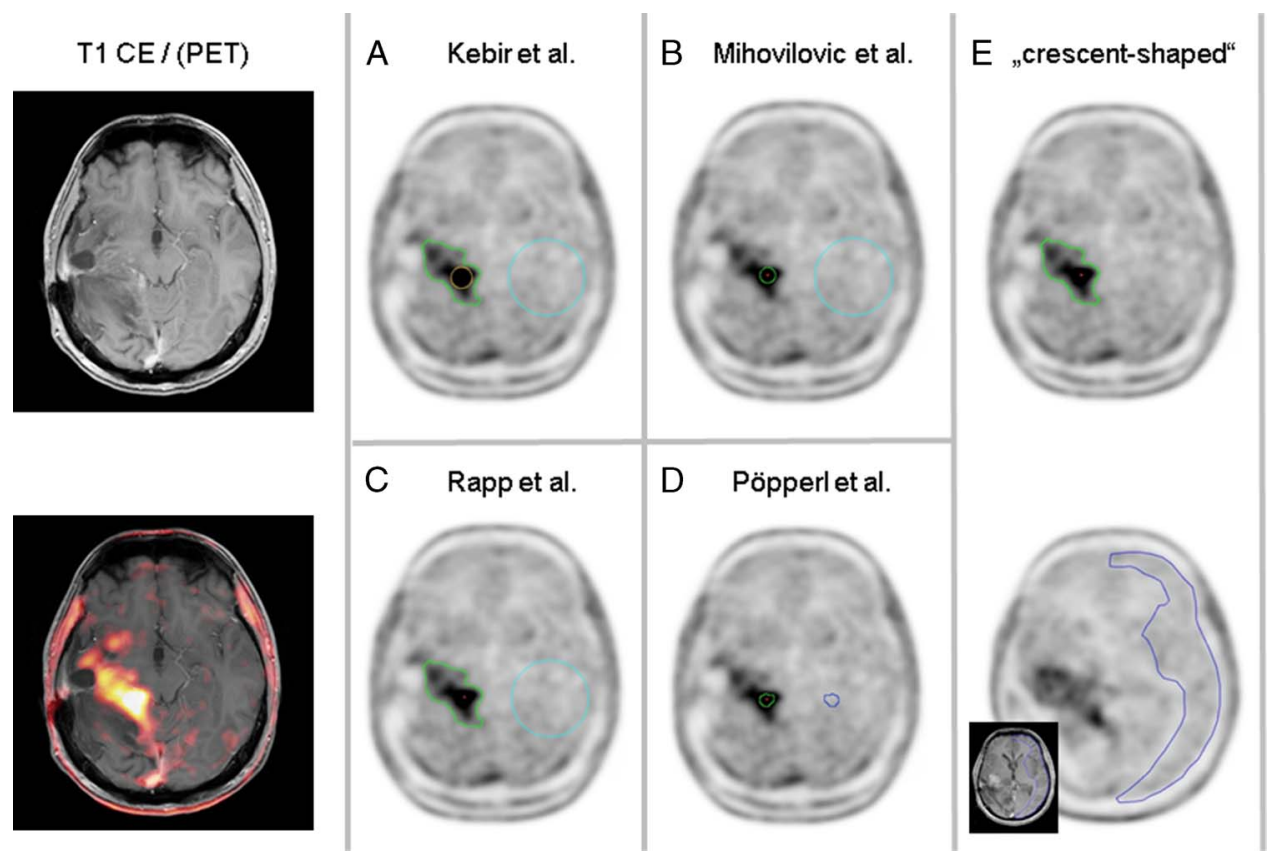

FIGURE 1. Example of target and reference region delineations of the different analytical approaches. Shown is an example of the different analytical approaches for tumor and background SUV derivation in patient 19. A, Kebir et al ${ }^{13}$ : background

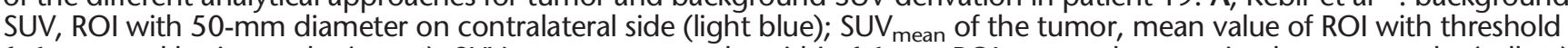
$1.6 \times$ normal brain uptake (green); $S U V_{16 \mathrm{~mm}}$, mean uptake within 16-mm ROI centered on maximal tumor uptake (yellow). B, Mihovilovic et $\mathrm{al}^{14}$ : background SUV, ROI with $50-\mathrm{mm}$ diameter on contralateral side (light blue); SUV $10 \mathrm{~mm}$ of the tumor,

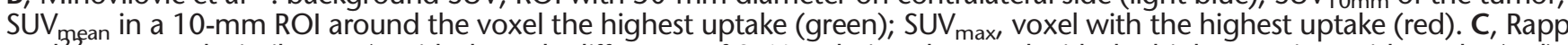
et $\mathrm{al}^{22}$ : approach similar to A), with the only difference of SUV $\max$ being the voxel with the highest amino acid uptake (red).

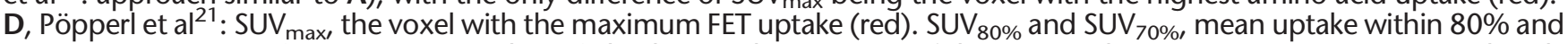
$70 \%$ isocontour ROIs (green, $80 \%$ ROI shown); background SUV, mean of the $70 \%$ and $80 \%$ isocontour ROI mirrored to the contralateral hemisphere (blue, $80 \%$ ROI shown). E, Crescent shaped: background SUV, mean uptake of crescent-shaped VOI positioned on 3 transaxial slices on the level of the basal ganglia (blue); tumor SUV mean (green) and SUV max (red), identical to Rapp et al. ${ }^{22}$ Additionally, a $90 \%$ isocontour covering the lesion on the transaxial slice with the highest uptake (SUV ${ }_{90 \%}$ ) was used (not shown). 


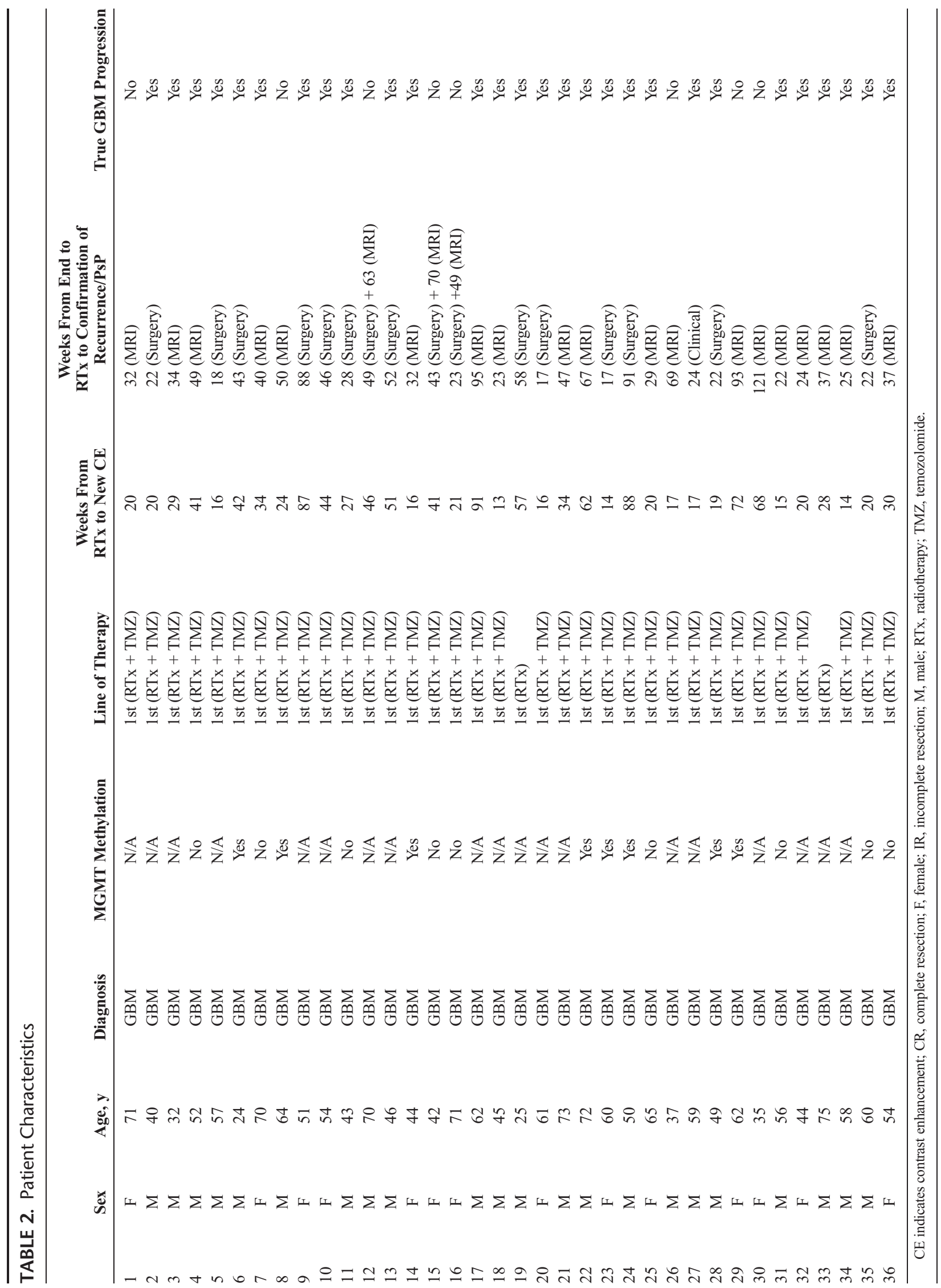


or after radiotherapy alone $(\mathrm{n}=2)$, respectively. Individual patient data are shown in Table 2.

\section{Diagnosis of True Tumor Progression Versus Late PsP}

Diagnosis of true tumor progression versus late PsP was established by histological analysis of surgical tumor samples in 16 of 36 patients and by clinical and radiological examination in the remainder. In total, true tumor progression was diagnosed in 28 of 36 cases and late PsP in the remaining 8 subjects (Fig. 2).

\section{Imaging Results}

All TBR measures were significantly higher in patients with true tumor progression as compared with late PsP regardless of the semiquantitative approach applied (all $P \leq 0.002$, respectively). Values for TBR obtained using the different analytical strategies are given in Table 3 (mean $\pm \mathrm{SD}$ ).

The ROC analysis yielded roughly comparable areas under the curve (AUCs) for each of the different approaches for the differentiation between true glioma progression and late $\mathrm{PsP}$, ranging from $0.80\left(\mathrm{TBR}_{\text {mean }}\right.$ derived according to Kebir et al, ${ }^{13}$ Rapp et $\left.\mathrm{al}^{22}\right)$ to $0.88\left(\mathrm{TBR}_{\text {mean } 80 \%}\right.$ derived according to Pöpperl et $\left.\mathrm{al}^{21}\right)$. The ROC AUC differences among the 10 methods were not statistically significant (all $P>0.05$; Table 4). However, it is interesting to note that TBR outcome measures relying on liberal tumor definitions (ie, $\mathrm{TBR}_{\text {mean }}$ using a TBR threshold $>1.6$ in the approaches by Kebir et $\mathrm{al}^{13}{ }^{13}$ Rapp et al, ${ }^{22}$ and crescent shaped; $\mathrm{AUC}=0.80-0.83$ ) or on fixed-size nonanatomical tumor definitions (ie, $\mathrm{TBR}_{16 \mathrm{~mm}}$ and $\mathrm{TBR}_{10 \mathrm{~mm}}$ by Kebir et $\mathrm{al}^{13}$ and Mihovilovic et $\mathrm{al}^{14} ; \mathrm{AUC}=0.81-0.82$ ) provided the lowest ROC AUC values. Compared with the aforementioned parameters and within each of the parameter sets relying on the same reference region, the diagnostic performance of $\mathrm{TBR}_{\max }$ was consistently higher (Rapp et al, ${ }^{22}$ Mihovilovic et al, ${ }^{14}$ and crescent shaped, AUC $=0.84-0.86$ ), which tended to be slightly outperformed by TBR measures relying on multiple voxels with the highest uptake $\left(\mathrm{TBR}_{70 \%}, \mathrm{TBR}_{80 \%}\right.$, and $\mathrm{TBR}_{90 \%}$ according to Pöpperl et $\mathrm{al}^{21}$ and the crescent-shaped method, ROC AUC $=0.85-0.88$ ).

Optimum cutoff values as well as the corresponding values for sensitivity and specificity for all contemplated TBR outcome measures are shown in Supplemental Table 1 (Supplemental Digital Content 1, http://links.lww.com/CNM/A202).

\section{DISCUSSION}

The value of ${ }^{18}$ F-FET PET as an easy-to-read and robust tool in glioma imaging is well acknowledged and has been demonstrated over many years. ${ }^{23,24}$ However, the methodology is yet to be harmonized. Depending on the history and preference of the respective clinical site, differences exist in imaging protocols (dynamic vs static acquisition) as well as definition of both the tumor and the normal brain reference regions. Previous studies have reported on the influence of reference region definition ${ }^{16}$ and data processing at different imaging centers. ${ }^{17}$ In the present study, we compared 10 different analytic approaches (ie, relying on 7 and 3 different methods for tumor and normal brain reference region definition, respectively) in the setting of suspected late PsP in GBM using static ${ }^{18}$ F-FET PET. In the present cohort, all approaches achieved roughly comparable performance in the differentiation of nonspecific treatment-related changes from true tumor progression with ROC AUC ranging between 0.80 and 0.88 , thus confirming the general suitability of ${ }^{18}$ F-FET PET for the definition of biologically active tumor.

Noteworthy, TBR outcome measures relying on liberal tumor definitions (ie, $\mathrm{TBR}_{\text {mean }}$ using a TBR threshold $>1.6$; $\mathrm{AUC}=0.80-0.83$ ) or on fixed-size nonanatomical tumor definitions (ie, $\mathrm{TBR}_{16 \mathrm{~mm}}$ and $\mathrm{TBR}_{10 \mathrm{~mm}}$; $\mathrm{AUC}=0.81-0.82$ ) yielded the lowest ROC AUC values observed. This may be explained by the fact that the aforementioned threshold was defined on primary brain tumors (low and high grade), ${ }^{4}$ while the distinction between true tumor progression and $\mathrm{PsP}$ is complicated by benign treatment-related changes with increased FET uptake (among others, including technical factors) that necessitate higher cutoff values. ${ }^{8,27}$ Likewise, fixed-size nonanatomical tumor definitions are expected to include nonneoplastic tissue like normal tissue, scar tissue or even cerebrospinal fluid spaces. This observation underlines that need for using suitable thresholds for a given clinical situation and/or proper anatomical tumor region definition.

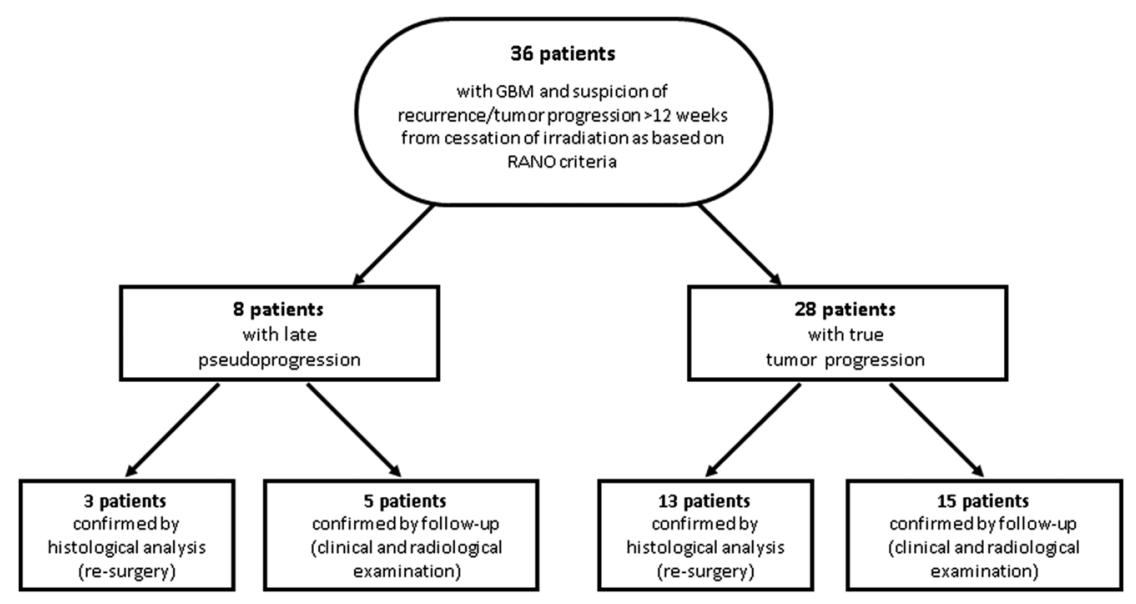

FIGURE 2. Flowchart of participant selection and outcomes. Between April 2010 and August 2016,36 consecutive patients (22 males and 14 females; aged 24-75 years; mean age, $54 \pm 14$ years) with a history of GBM were included. All patients had previously undergone external beam radiation (end of radiation $>12$ weeks prior to presentation in all cases) and were now referred to PET due to MRI-based suspicion of recurrence/progression of GBM according to RANO working group criteria. Diagnosis of true tumor progression versus late PsP was established by histological analysis of surgical tumor samples in 16 of 36 patients and by clinical and radiological examination in the remainder (20/36). In total, true tumor progression was diagnosed in 28 of 36 cases and late PsP in the remaining 8 subjects. PET robustly differentiated late PsP from true tumor recurrence with ROC AUCs ranging from 0.80 to 088. 
TABLE 3. Results of Regional Analyses (Mean \pm SD Over All Patients)

\begin{tabular}{|c|c|c|c|}
\hline & Parameter & $\operatorname{PsP}(n=8)$ & Tumor Progression $(\mathrm{n}=\mathbf{2 8})$ \\
\hline \multirow[t]{2}{*}{ Kebir et al ${ }^{13}$} & $\mathrm{TBR}_{\text {mean }}^{1}$ & $2.08 \pm 0.18$ & $2.37 \pm 0.30$ \\
\hline & $\mathrm{TBR}_{16 \mathrm{~mm}}$ & $2.31 \pm 0.35$ & $2.89 \pm 0.53$ \\
\hline \multirow[t]{2}{*}{ Rapp et $\mathrm{al}^{22}$} & $\mathrm{TBR}_{\text {mean }}^{1}$ & $2.08 \pm 0.18$ & $2.37 \pm 0.30$ \\
\hline & $\mathrm{TBR}_{\max }^{2}$ & $3.22 \pm 0.62$ & $4.43 \pm 1.01$ \\
\hline \multirow[t]{2}{*}{ Mihovilovic et $\mathrm{al}^{14}$} & $\mathrm{TBR}_{10 \mathrm{~mm}}$ & $2.70 \pm 0.53$ & $3.56 \pm 0.79$ \\
\hline & $\mathrm{TBR}_{\max }^{2}$ & $3.22 \pm 0.62$ & $4.43 \pm 1.01$ \\
\hline \multirow[t]{3}{*}{ Pöpperl et $\mathrm{al}^{21}$} & $\mathrm{TBR}_{70 \%}$ & $2.47 \pm 0.37$ & $3.48 \pm 0.81$ \\
\hline & $\mathrm{TBR}_{80 \%}$ & $2.72 \pm 0.39$ & $3.77 \pm 0.86$ \\
\hline & $\mathrm{TBR}_{\max }$ & $3.11 \pm 0.47$ & $4.27 \pm 0.95$ \\
\hline \multirow[t]{3}{*}{ "Crescent shaped" } & $\mathrm{TBR}_{\text {mean }}$ & $2.06 \pm 0.15$ & $2.36 \pm 0.26$ \\
\hline & $\mathrm{TBR}_{90 \%}$ & $2.84 \pm 0.33$ & $3.86 \pm 0.97$ \\
\hline & $\mathrm{TBR}_{\max }$ & $3.01 \pm 0.38$ & $4.09 \pm 1.00$ \\
\hline
\end{tabular}

TBR is SUV of target region divided by SUV of reference region; for region definition, see Table 1.

Superscripts 1 and 2 denote pairs of identical analytical approaches.

Thus, given the use of different scanners for data acquisition as well as different reconstruction software and parameters, cutoffs for the definition of vital tumor need to be confirmed individually for each imaging center and clinical situation.

In line with this, approaches utilizing $\mathrm{SUV}_{\max }$ (ROC AUC $=0.84-0.86)$ as an imaging analog of a "punch biopsy" targeting the most suspicious lesion part were consistently superior to aforementioned approaches, although not reaching statistical significance. However, methods relying on $\mathrm{SUV}_{\max }$ are generally more susceptible to the confounding influence of PET system performance, image reconstruction, and noise (eg, spatial resolution, reconstruction-induced noise enhancement, postfiltering) than any average-based method employing larger regions. As a consequence, we and others ${ }^{21}$ also used isocontour approaches including multiple voxels with the highest ${ }^{18} \mathrm{~F}$-FET uptake. In fact, in direct comparison to $\mathrm{TBR}_{\max }, \mathrm{TBR}_{70 \%}, \mathrm{TBR}_{80 \%}$, and $\mathrm{TBR}_{90 \%}$ tended to perform slightly better (ROC AUC $=0.85-0.88$ ).

It is important to note that the definition of the normal brain reference region is just as important as the definition of the tumor target regions. In a recent publication, Unterrainer et $\mathrm{al}^{16}$ discussed the need of a consistent method of background activity assessment and proposed a crescent-shaped background VOI as a reproducible approach for methodological standardization. ${ }^{16}$ We also employ a similar approach in clinical routine using clear instructions for definition of a large, anatomically defined reference region (see Materials and Methods), which usually also incorporates coregistration with MRI (if available). This is done to reduce noise, increase reproducibility, and avoid potential pitfalls of reference region definition (eg, inclusion of structural changes due to atrophy, trauma, or ische$\mathrm{mia}$ ) that can hardly be avoided when using fixed-size nonanatomical reference regions or strictly mirrored tumor regions as reference regions. In addition, a method for definition of tumor and normal brain tissue should be advocated for all clinical situations.

Although various approaches for the differentiation of late PsP from true tumor progression proved feasible in the current study, harmonization of PET analysis is of high relevance, in particular with regard to future prospective, multicenter trials to foster evidence of the added value of amino acid-based PET in glioma or when using ${ }^{18} \mathrm{~F}$-FET PET for treatment decisions and planning. Future guidelines should recommend standard approaches for the imaging protocols (eg, dynamic vs static acquisition, reconstruction methods, use of resolution recovery, etc) and analysis method (method of region definition) to facilitate comparisons between different sites, reduce sources of errors, and eventually establish an optimal study setup for future research. Based on the present results and aforementioned theoretical reasoning, the use of an isocontour including multiple voxels with the highest uptake (eg, $\mathrm{SUV}_{80 \%}$ or $\mathrm{SUV}_{90 \%}$ ) and a large, anatomically defined reference region (eg, crescent shaped) seems to be particularly advisable. The latter recommendation may apply to clinical settings analogous not only to the present setting (ie, decision on the presence or absence of viable tumor tissue) but also to the delineation of tumor extent.

Limitations of this study include its small sample size and its retrospective nature. Additionally, no dynamic acquisitions were performed that may provide valuable diagnostic information. ${ }^{28-30}$ Noteworthy, the TBR values (especially $\mathrm{TBR}_{\max }$ ) in our cohort — while

TABLE 4. ROC AUCs of the TBR Outcome Measures

AUC (95\% Confidence Interval)

\begin{tabular}{lc}
\hline Kebir et al $^{13}$ & \\
$\mathrm{TBR}_{\text {mean }}^{1}$ & $0.80(0.64-0.95)$ \\
$\mathrm{TBR}_{16 \mathrm{~mm}}$ & $0.82(0.67-0.97)$ \\
Rapp et al $^{22}$ & \\
$\mathrm{TBR}_{\text {mean }}^{1}$ & $0.80(0.64-0.95)$ \\
$\mathrm{TBR}_{\text {max }}^{2}$ & $0.86(0.72-1.00)$ \\
Mihovilovic et al $^{14}$ & \\
$\mathrm{TBR}_{10 \mathrm{~mm}}$ & $0.81(0.65-0.97)$ \\
$\mathrm{TBR}_{\text {max }}^{2}$ & $0.86(0.72-1.00)$ \\
Pöpperl et al $^{21}$ & \\
$\mathrm{TBR}_{70 \%}$ & $0.87(0.75-0.99)$ \\
$\mathrm{TBR}_{80 \%}$ & $0.88(0.76-0.99)$ \\
$\mathrm{TBR}_{\text {max }}$ & $0.86(0.73-0.99)$ \\
"Crescent shaped” & \\
$\mathrm{TBR}_{\text {mean }}$ & $0.83(0.69-0.97)$ \\
$\mathrm{TBR}_{90 \%}$ & $0.85(0.72-0.97)$ \\
$\mathrm{TBR}_{\text {max }}$ & $0.84(0.71-0.97)$
\end{tabular}

All approaches yielded comparable AUC with all $P>0.05$, respectively. TBR is SUV of target region divided by SUV of reference region; for region definition, see Table 1.

Superscripts 1 and 2 denote pairs of identical analytical approaches. 
accurately delineating PsP from true tumor progression - turned out to be considerably higher in our cohort than those previously published by other groups. ${ }^{13}$ While a potential influence from treatment-related changes with associated nonspecific ${ }^{18}$ F-FET uptake cannot be excluded, a major contribution to this difference might be assigned to resolution recovery applied during iterative PET reconstruction (TrueX algorithm). In addition to the commonly used correction factors, TrueX, on the one hand, improves the visual appearance of the PET image but, on the other hand, results in an overestimation of the maximum activity observed (especially in small VOIs $<12 \mathrm{~mL}^{31}$ ). Thus, the cutoff values given in Supplemental Table 1 (Supplemental Digital Content 1, http://links.lww.com/CNM/A202) cannot be easily transferred to other settings.

\section{CONCLUSIONS}

${ }^{18}$ F-FET PET is a robust tool for detection of late PsP in GBM, irrespective of the analytical approach. However, methodological standardization and harmonization to ensure comparability between different centers would be highly desirable.

\section{REFERENCES}

1. Chen W. Clinical applications of PET in brain tumors. J Nucl Med. 2007;48: 1468-1481.

2. Pöpperl G, Kreth FW, Herms J, et al. Analysis of ${ }^{18}$ F-FET PET for grading of recurrent gliomas: is evaluation of uptake kinetics superior to standard methods? J Nucl Med. 2006;47:393-403.

3. Albert NL, Winkelmann I, Suchorska B, et al. Early static (18)F-FET-PET scans have a higher accuracy for glioma grading than the standard 20-40 min scans. Eur J Nucl Med Mol Imaging. 2016;43:1105-1114.

4. Pauleit D, Floeth F, Hamacher K, et al. O-(2-[ $\left[{ }^{18} \mathrm{~F}\right]$ fluoroethyl)-L-tyrosine PET combined with MRI improves the diagnostic assessment of cerebral gliomas. Brain. 2005;128:678-687.

5. Pauleit D, Stoffels G, Bachofner A, et al. Comparison of (18)F-FET and (18)F-FDG PET in brain tumors. Nucl Med Biol. 2009;36:779-787.

6. Plotkin M, Blechschmidt C, Auf G, et al. Comparison of F-18 FET-PET with F-18 FDG-PET for biopsy planning of non-contrast-enhancing gliomas. Eur Radiol. 2010;20:2496-2502.

7. Jansen NL, Suchorska B, Wenter V, et al. Prognostic significance of dynamic ${ }^{18}$ F-FET PET in newly diagnosed astrocytic high-grade glioma. $J$ Nucl Med. 2015;56:9-15.

8. Suchorska B, Jansen NL, Linn J, et al. Biological tumor volume in ${ }^{18}$ FET-PET before radiochemotherapy correlates with survival in GBM. Neurology. 2015; 84:710-719.

9. Unterrainer M, Schweisthal F, Suchorska B, et al. Serial ${ }^{18}$ F-FET PET imaging of primarily ${ }^{18} \mathrm{~F}$-FET-negative glioma: does it make sense? J Nucl Med. 2016;57:1177-1182.

10. Galldiks N, Langen KJ, Holy R, et al. Assessment of treatment response in patients with glioblastoma using O-(2- ${ }^{18} \mathrm{~F}$-fluoroethyl)-L-tyrosine PET in comparison to MRI. J Nucl Med. 2012;53:1048-1057.

11. Hutterer M, Nowosielski M, Putzer D, et al. O-(2- ${ }^{18}$ F-fluoroethyl)-L-tyrosine PET predicts failure of antiangiogenic treatment in patients with recurrent high-grade glioma. J Nucl Med. 2011;52:856-864.

12. Ceccon G, Lazaridis L, Stoffels G, et al. Use of FET PET in glioblastoma patients undergoing neurooncological treatment including tumour-treating fields: initial experience. Eur J Nucl Med Mol Imaging. 2018.
13. Kebir S, Fimmers R, Galldiks N, et al. Late pseudoprogression in glioblastoma: diagnostic value of dynamic O-(2-[ $\left.{ }^{18} \mathrm{~F}\right]$ fluoroethyl)-L-tyrosine PET Clin Cancer Res. 2016;22:2190-2196.

14. Mihovilovic MI, Kertels O, Hanscheid H, et al. O-(2-((18)F)fluoroethyl)-L-tyrosine PET for the differentiation of tumour recurrence from late pseudoprogression in glioblastoma. J Neurol Neurosurg Psychiatry. 2019;90:238-239.

15. Galldiks N, Dunkl V, Stoffels G, et al. Diagnosis of pseudoprogression in patients with glioblastoma using O- $\left(2-\left[{ }^{18} \mathrm{~F}\right]\right.$ fluoroethyl $)$-L-tyrosine PET. Eur J Nucl Med Mol Imaging. 2015;42:685-695.

16. Unterrainer M, Vettermann F, Brendel M, et al. Towards standardization of ${ }^{18}$ F-FET PET imaging: do we need a consistent method of background activity assessment? EJNMMI Res. 2017;7:48.

17. Filss CP, Albert NL, Böning G, et al. O-(2-[ $\left[{ }^{18} \mathrm{~F}\right]$ fluoroethyl)-L-tyrosine PET in gliomas: influence of data processing in different centres. EJNMMI Res. 2017;7:64.

18. Oehlke O, Mix M, Graf E, et al. Amino-acid PET versus MRI guided re-irradiation in patients with recurrent glioblastoma multiforme (GLIAA) - protocol of a randomized phase II trial (NOA 10/ARO 2013-1). BMC Cancer. 2016;16:769.

19. Lapa C, Linsenmann T, Monoranu CM, et al. Comparison of the amino acid tracers ${ }^{18}$ F-FET and ${ }^{18}$ F-DOPA in high-grade glioma patients. $J$ Nucl Med. 2014;55:1611-1616

20. Langen KJ, Bartenstein P, Boecker H, et al. German guidelines for brain tumour imaging by PET and SPECT using labelled amino acids. Nuklearmedizin. 2011;50:167-173.

21. Pöpperl G, Götz C, Rachinger W, et al. Value of O-(2-[ $\left.{ }^{18} \mathrm{~F}\right]$ fluoroethyl)-L-tyrosine PET for the diagnosis of recurrent glioma. Eur J Nucl Med Mol Imaging. 2004; $31: 1464-1470$

22. Rapp M, Heinzel A, Galldiks N, et al. Diagnostic performance of ${ }^{18} \mathrm{~F}-\mathrm{FET}$ PET in newly diagnosed cerebral lesions suggestive of glioma. $J \mathrm{Nucl}$ Med. 2013;54:229-235.

23. Albert NL, Weller M, Suchorska B, et al. Response Assessment in NeuroOncology working group and European Association for Neuro-Oncology recommendations for the clinical use of PET imaging in gliomas. Neuro Oncol. 2016;18:1199-1208.

24. Langen KJ, Galldiks N, Hattingen E, et al. Advances in neuro-oncology imaging. Nat Rev Neurol. 2017;13:279-289.

25. DeLong ER, DeLong DM, Clarke-Pearson DL. Comparing the areas under two or more correlated receiver operating characteristic curves: a nonparametric approach. Biometrics. 1988;44:837-845.

26. Stupp R, Mason WP, van den Bent MJ, et al; European Organisation for Research and Treatment of Cancer Brain Tumor and Radiotherapy Groups; National Cancer Institute of Canada Clinical Trials Group. Radiotherapy plus concomitant and adjuvant temozolomide for glioblastoma. $N$ Engl J Med. 2005;352:987-996.

27. Jansen NL, Graute V, Armbruster L, et al. MRI-suspected low-grade glioma: is there a need to perform dynamic FET PET? Eur J Nucl Med Mol Imaging. 2012;39:1021-1029.

28. Popperl G, Kreth FW, Mehrkens JH, et al. FET PET for the evaluation of untreated gliomas: correlation of FET uptake and uptake kinetics with tumour grading. Eur J Nucl Med Mol Imaging. 2007;34:1933-1942.

29. Kunz M, Albert NL, Unterrainer M, et al. Dynamic ${ }^{18}$ F-FET PET is a powerful imaging biomarker in gadolinium-negative gliomas. Neuro Oncol. 2018.

30. Suchorska B, Giese A, Biczok A, et al. Identification of time-to-peak on dynamic ${ }^{18}$ F-FET-PET as a prognostic marker specifically in IDH1/2 mutant diffuse astrocytoma. Neuro Oncol. 2018;20:279-288.

31. Knausl B, Hirtl A, Dobrozemsky G, et al. PET based volume segmentation with emphasis on the iterative TrueX algorithm. Z Med Phys. 2012;22: 29-39. 